\title{
PLATINE: DVB-S2/RCS Enhanced Testbed for Next Generation Satellite Networks
}

C. Baudoin ${ }^{1}$, M. Dervin ${ }^{1}$, P. Berthou ${ }^{2}$, T. Gayraud ${ }^{2}$, F. Nivor ${ }^{2}$, B. Jacquemin ${ }^{2}$, D. Barvaux ${ }^{3}$ and J. Nicol ${ }^{3}$

${ }^{1}$ Thales Alenia Space, 26 Avenue JF Champollion, BP 33787, 31037 Toulouse Cedex 1, France,

Phone: + 330534353637

\{Cedric.Baudoin, Mathieu Dervin\}@thalesaleniaspace.com ;

${ }^{2}$ LAAS-CNRS, 7 avenue du Colonel Roche - 31077 Toulouse Cedex 4, France Phone: +330561336200

\{Berthou, Gayraud, Nivor, Jacquemin $\}$ laas.fr

${ }^{3}$ B2i, 1 avenue de l'Europe, 31400 Toulouse, France

Phone : +330561003080

\{Didier.Barvaux, Julien.Nicol\}@b2i-toulouse.com

\section{Abstract}

Emulation is a cost effective and efficient tool to perform performances evaluation and innovative access and network techniques validation. Its ability to interconnect real equipments with real applications provides excellent demonstrations means. The main problem is to overcome the emulation weakness which is the accuracy of the model reproducing the systems to be evaluated. Owing to its modular design and implementation, the PLATINE satellite emulation platform, presented in this paper, is able to emulate a complete DVB-RCS (Digital Video Broadcasting Return Channel via Satellite)- DVB-S2 (Digital Video Broadcasting - Second ) system in a realistic and flexible way. It is possible to configure the platform to emulate a transparent DVBRCS system dimensioned around a single Hub, or to emulate a system using a regenerative satellite with an on-board switching matrix. Different DVB-RCS protocol stacks are implemented, and the adaptive physical layer is emulated in real time thanks to pre-calculated DRA schemes and MODCOD files. A DiffServ-like QoS Architecture that couples MAC and IP-Layer QoS mechanisms and Layer 2 security framework are currently under development. At the network side, IPv4 and IPv6 are fully supported as well as IPv6 mobility and dynamic multicast. In this paper, we mainly focus on the emulation platform and the tools developed to help the performance analysis of the emulated system. 


\section{Introduction}

Evaluating performances over real satellite data links or network is often costly, even impossible for systems in development phase. Simulation and emulation both provide the opportunity to evaluate performances, at low cost, on more or less realistic systems. When simulation needs a complete modeling of the systems from applications to physical network and operates in virtual time, emulation is more demonstrative since real applications can be deployed over the model describing transfer characteristics, delay and error behavior for instance.

The IST SATSIX [1] project relies on these different methods of evaluation and validation, with the design and development of simulations, emulation and trials. The emulation testbed relies on the satellite emulation platform (PLATINE) [7] formerly developed within the frame of the IST SATIP6 [2] project. It aims to demonstrate the network and application services integration on next generation satellite systems and the possibility to interoperate with terrestrial networks. With regards to the previous release, PLATINE includes a DVB-RCS and DVB-S2 emulation, with ULE/MPEG2-TS and AAL5/ATM stacks together with the adaptive physical layer simulation and the associated radio resources management (RRM). A complete QoS architecture mixing SIP proxies, IP/MAC scheduling and cross layer techniques is available. Finally, a layer 2 security framework will be implemented. Moreover, various network techniques, such as IPv6 mobility, dynamic multicast and its interaction with mobility thanks to MLD proxy are carried on in the frame of SATSIX, making our platform a good opportunity to test new schemes, protocols and services for next generation satellite networks.

This paper first briefly sums up the main functionalities of DVB-RCS satellite systems. The second section depicts the design and the implementation of our full DVBS2/RCS emulated satellite platform. It mainly focuses on the satellite Physical and Data Link layers techniques and the final QoS architecture that was retained to provide differentiated QoS on a satellite network. The final section presents the environment tools used to collect statistics, events and errors and to control the testbed in a distributed way.

\section{Introduction to DVB-RCS systems}

\subsection{PLATINE objectives}

The Satellite network emulation testbed (PLATINE) has been initially developed within the frame of the SATIP6 IST project. It is compliant with the architecture adopted within the ETSI BSM [4] group and the DVB-RCS and DVB-S2 standards. Different system scenarios can be handled by the emulation testbed. The first scenario, shown in Figure 1, consists in a geostationary satellite network with onboard switching capabilities and DVB-RCS uplinks and DVB-S2 downlinks. The satellite is regenerative meaning that only a single hop is needed to interconnect two end users. Satellite Terminals (RCST) provide single PC or LANs with the access to the network, while Gateways (GWs) allow the connection with Internet core networks. The second scenario is related to a more classical transparent satellite providing network access to users. RCST can access a gateway. STs and GWs are boundary devices between the satellite and terrestrial links and play an important role in access to satellite resources and hence in QoS provisioning. Both devices implement IP routing and have an IP interface on the satellite segment, as IP serves as a common denominator between the satellite and terrestrial networks. That is to say that Satellite Network is considered as a special link from a classical network point of view.

\section{Fig. 1}


Figure 1 depicts the satellite network architecture emulated by the PLATINE platform. On the left is represented the end-user side of the platform. On the right is shown the provider/enterprise/Internet side of the platform. We distinguish also between the satellite network side (in the middle) and the IP network sides (on left and right ends), interconnected by RCSTs.

Three main components have to be distinguished in the satellite network side (middle): the Satellite, the Return Channel Satellite Terminals (RCST) and the Network Control Center (NCC).

The role of the different network elements (NCC, RCST) is explained in a brief introduction to the main ETSI recommendations related to QoS in satellite.

\subsection{Introduction to DVB-RCS Architecture}

Initiated in 1993, the international European DVB Project published, in the endnineties, a family of digital transmission specifications, based upon MPEG-2 (Motion Picture Expert Group) video compression and transmission techniques. In each specification, data are thus transported within MPEG-2 transport streams (MPEG2-TS) which are identified through DVB Service Information Tables. Adapted for satellite systems, DVB-S defines one of the most widespread formats used for Digital TV over the last years and still nowadays. However, DVB-S Satellite Terminals can then only receive frames from the satellite. The need for a return link rapidly becomes essential so as to support emerging Internet services via satellite . DVB-RCS, published in March 2000, provides full bidirectionnal satellite architecture. Concerning IP encapsulation, various schemes already exist and are still being specified for the forward and return link.

The latest version of DVB-RCS and DVB-S2 introduces Fade Mitigation Techniques (FMT) to mitigate propagation effects on communication links. It consists in adapting the waveform, either by adapting the coding and/or modulation scheme or by reducing the data rate (on the uplink) to cope with systems constraints (see Figure 2).

The return link access scheme in DVB-S/RCS systems is MF-TDMA. The return link is segmented into portions of time and frequency ("superframes"), each of which is divided into timeslots ("bursts") of either fixed or variable durations and bandwidths during which STs are able to transmit MPEG2-TS packets or ATM cells.

The entire satellite system control, especially STs synchronization and resource allocation, is performed by the NCC. It periodically broadcasts a signaling frame, the TBTP (Terminal Burst Time Plan), which updates the timeslot allocation within a superframe between every competing ST. This allocation can be dynamically modified on STs demand thanks to a bandwidth on demand protocol called Demand Assignment Multiple Access (DAMA). It supplements the STs with the ability to frequently request capacities that fit their current respective traffic load to the NCC. However the DAMA request/assignment cycle exhibits a non negligible latency and additional delays that cannot always match interactivity requirements of multimedia services. In order to maximize satellite resource use and meet multimedia requirements, the DVB-RCS norm discriminates RCST capacity requests into 4 categories:

- Continuous Rate Assignment (CRA): fixed slots are assigned in each MFTDMA frame for the whole duration of a RCST connection

- Rate-Based Dynamic Capacity (RBDC): a dynamic rate capacity (in slots/frame) granted in response to explicit RCST requests

- Volume-Based Dynamic Capacity (VBDC): a dynamic cumulative volume capacity (in slots), granted in response to explicit RCST requests

- Free Capacity Allocation (FCA), which is assigned to STs on an "as available" basis from unused capacity.

The standard, after defining separate MAC traffic priority queues (Real-Time, Variable Rate and Jitter-Tolerant priorities), suggests a requesting strategy for each of them, that is to say a relevant mapping between traffic and request categories. Any given RCST can be assigned one or a mix of the four capacity types. In general, higher priority 
classes of service (e.g. IP DiffServ EF and AF classes) are associated with guaranteed capacity (CRA, RBDC), while lower priority classes (e.g. Best Effort) are predominantly given best effort capacity (VBDC, FCA).

\subsection{Experimental platform}

The testbed we have built is able to emulate a complex scenario for next generation satellite network as presented in figure 1. Each network element involved in the satellite network is emulated in our platform on a dedicated node. In fact, 3 users LAN composed of two nodes (standard Linux systems) are connected to the emulated satellite network with 3 RCST that implement an almost complete DVB-S2/RCS stack. The satellite core network is emulated thanks to the Satellite Emulator (SE) as link emulator and the Network Control Center (NCC) for bandwidth management (DAMA). 9 computers are used as described in Figure 3.

\section{Fig. 3}

We first describe the protocol stacks implementation, the satellite carrier emulation, the MAC layer implementation and then the QoS architecture.

\section{The PLATINE architecture implementation}

\subsection{The Satellite System Emulation}

In order to have the most modular platform and so preserve room for future evolution (GSE), stringent requirements were fixed before the development phase.

At first, the emulation testbed takes advantage of a linux system (Fedora Core 5) which natively supports IPv6 and a wide panel of IPv6 applications (Apache as HTTP Server, Mozilla as HTTP Client, Vsftpd as FTP Server, Gnomemeeting for Videoconferencing, VideoLanClient for Videostreaming), as well as advanced network and QoS features.

The core architecture of PLATINE relies on a derived GPL C++ runtime called Margouilla [3], that provides platform independent messaging and synchronization toolkit, bloc management, a set of common blocs ready to use (IP/ATM/Ethernet layers...) and various utilities packages such as configuration file and logging mechanisms for error and debug messages. The final PLATINE protocol stack is detailed in Figure 4.

\section{Fig. 4}

The blocks that were developed within the Margouilla runtime are:

- The satellite carrier package is responsible for the different satellite carriers emulation on top of Ethernet (DVB-RCS, DVB-S2 and Signaling Channels) and the simulation of typical satellite bit errors and delay.

- The DVB-S2/RCS package implements a framing structure compliant with the DVB-S2/RCS standards. and fills DVB-RCS frames with upper layer packets (ATM or MPEG2-TS) coming from the ENCAP bloc layer. In order to achieve proper QoS, this layer manages synchronization and queues according to the authorizations a DAMA algorithm delivers.

- The DAMA package implements the DAMA algorithms used to manage the satellite resources allocation at layer 2 taking into account adaptive physical layer information. 
- The ENCAP package implements AAL5 and ULE encapsulation schemes, and is in charge of the segmentation and reassembly functionalities (ATM or MPEG2TS).

- The IP QoS package implements common mechanisms to enable differentiation at this level. It mostly relies on QoS services offered by Linux kernel through the Traffic Conditioning (TC) tool, retrieves incoming packets from IP network with their associated tag and forwards them to the lower layer.

A detailed description of the way the different layers are emulated is done through the following paragraphs.

\subsubsection{The satellite carrier emulation}

The satellite carrier emulation is designed to operate on top of Ethernet frames and for each satellite channel corresponds an Ethernet multicast address. Ethernet was chosen for its native broadcast abilities (like a spot) and also for its high bandwidth capacities. For each spot, different logical channels are defined to transport data on the air interface depending on their type (data, logon , control, ...). This component is also in charge of dropping frame not belonging to the node or received with a MODCOD more efficient than it can receive.

\subsubsection{The Satellite Link Emulation}

The satellite emulator (SE) can act as a transparent or a regenerative satellite. It is able to emulate spot switching and signal format conversion. Indeed, the regenerative satellite with an onboard switching matrix processes DVB-RCS frames, switches ATM cells or MPEG2-TS received from the DVB-RCS frames, encapsulates ATM cells into MPEG2TS frames (in the ATM case) and finally forwards them within DVB-S2 frames. The switching mechanism is modular and can manage either ATM cells or MPEG2-TS frames. The switching tables could be updated by appropriated control message. Currently, the ATM and MPEG tables are allocated statically.

What is more, the main functionality of the SE is to emulate its satellite link, modelling configurable delay and jitter. In addition to simulate delay, error model can be introduced, either using precalculated BER files or statistical laws.

\subsubsection{Medium Access Control}

Due to hardware limitation and OS constraints, RCST and NCC components are synchronized on uplink frame and thus do not handle time slot synchronization. The precision of the emulation is therefore at best equal to one frame duration.

The emulation of the adaptive physical layer is done thanks to corresponding simulations performed in SATSIX the aim of which is to generate spatial rain patterns.

First of all, rain cells are spatially modeled by circular patterns regarding the rain rate parameter. Then, the circular hypothesis is extended to the rain attenuation, and to the allocated DRA schemes. Finally, Spatial rain patterns are generated from the DRA sequences time, issued from FMT simulations taking as an input simulated rain attenuation time series for an uplink availability of $99.9 \%$ of the time.

The result example for a spotbeam is shown in Figures 5 and 6 with 20 rain cells with $5 \mathrm{~dB}$ max. attenuation, and 4 cells with $11 \mathrm{~dB}$ max. attenuation moving across the beam and their impact on DRA schemes allowed for RCST.

\section{Fig. 5 \\ Fig. 6}


The frame synchronization is carried out by emitting Ethernet frames at fixed instant on all RCST (super frame tick) and then internally by awaking processes each frame ticks (50ms) to send already queued packet. The DVB-RCS allocation scheme is repeated for all the frames composing the superframe.

The allocation procedure (DAMA algorithm) implementation in the demonstrator is described below:

- At log-on RCSTs request a fixed bandwidth (CRA) for MAC "real-time (RT)" traffic. This allocation can be updated thanks to the dynamic QoS architecture using SIP proxy information.

- Then RCSTs is mainly in charge of the capacity request calculation that aims at achieving full link utilization thanks to a multi-MRC approach.

- Each Capacity Request (CR) is sent on a SYNC slot basis. Each CR is then delayed by the satellite emulator and reach NCC $250 \mathrm{~ms} \pm 10 \mathrm{~ms}$ after. CRs are processed by NCC upon arrival.

- NCC computes allocations at the beginning of each super frame according to an internal SACT table based on CR received. The DAMA algorithm takes into account DRA scheme of each RCST to constitute the frame structure. FCA based on weighted fair queuing scheduler can also been applied. Authorizations are sent back to RCSTs using a TBTP table.

- Upon reception of a TBTP, allocations are stored by RCST and used in the next frame.

\subsection{IP encapsulation}

This section deals with the transport of IP datagrams over the PLATINE emulation platform. The following paragraphs describe how IP encapsulation is handled by the platform components.

The ENCAP package is in charge of the IP encapsulation and segmentation/reassembly functionality. As presented in Figure 4, the ENCAP package is used by the RCST and GW components to send or receive IP datagrams through the satellite link. When the SE emulates a regenerative satellite, the OBP can manage two different encapsulation schemes for return and forward links thanks to the ENCAP package.

Various IP encapsulation schemes already exist or are still being specified for the forward and return link of DVB-RCS satellite system. The ENCAP package is designed to be modular, so that new encapsulation schemes can be easily added to the emulation testbed. The encapsulation schemes for forward and return links are chosen at simulation startup.

The ENCAP package currently implements AAL5 and ULE encapsulation schemes, as well as ATM and MPEG2-TS segmentation/reassembly mechanisms. The layer 2 security framework extends the ULE encapsulation scheme to add integrity and encryption functionalities.

The AAL5/ATM encapsulation and segmentation/reassembly scheme is presented in Figure 7. The ULE/MPEG2-TS mechanism defined in [9] is briefly described in Figure 8.

\section{Fig. 7}

Fig. 8 


\subsection{The QoS Architecture}

As presented in the firsts section, our platform implements a QoS architecture compliant with the ETSI Broadband Satellite Multimedia (BSM) group. This architecture links the MAC layer with the IP Layer to ensure the QoS continuity in the satellite protocol stack.

\subsubsection{MAC Layer}

Three MAC queues are available in the PLATINE system, one to satisfy a "real-time (RT)" service, one to provide a "non real time (nRT)" service and the last one to handle "best effort (BE)" service. The association between MAC queues and allocation categories is fully configurable. So the RT queue can be only served by Continuous Rate Assignment (CRA) or can also request additional capacity using RBDC requests. In the same way, the non real-time and best effort queues can be served either using RBDC or VBDC requests. Anyhow, allocation provided by the NCC (using CRA, RBDC, VBDC or FCA) is not affected to any specific queues, and is used to empty MAC queues in priority order.

\subsubsection{IP Layer}

The core of the IP QoS processing is performed by the HDLB scheduler which is based on the Linux HTB policy [8], but uses the Dual Leaky Bucket Filter algorithm to shape traffic (see Figure 9). It is mainly designed as a kernel module. It can be controlled in user space through the «TC » tool. The link between TC and the HDLB module is provided by a rtnetlink socket.

\section{Fig. 9}

Rtnetlink allows the kernel's routing tables to be read and altered. It is used within the kernel to communicate between various subsystems, and for communication with userspace programs. Network routes, IP addresses, link parameters, neighbor setups, queueing disciplines, traffic classes and packet classifiers may all be controlled through NETLINK_ROUTE sockets. It is based on netlink messages.

TC presents interfaces to attach or remove disciplines to qdiscs, and create or destroy classes of traffic. It creates messages to be sent to the rtnetlink socket. Platine uses a special version of TC adding HDLB classes compatibility. On the other side, the kernel module retrieves TC's commands and parameters and acts consequently. To get along, both TC and HDLB module use identical data structures. When HDLB module is first loaded into the kernel, the module registers the qdisc and class operations it is able to perform and that can be called from the user space. Figure 10 depicts an overview of the HDLB scheme.

\section{Fig. 10}

The IP Layer uses the following hierarchy, with basically 3 DiffServ categories (EF, $\mathrm{AF} 31$ to AF33, and BE) the parameters of which are dynamically updated by the QoS server according to QoS agent or SIP proxy information.

\subsubsection{Cross Layer mechanism}

The BSM group recommends cross layer mechanisms to enhance the adaptation between the IP and the MAC layers. The output throughput of the IP scheduler is adapted to the MAC output throughput thanks to feedback information. This scheme allows to optimize the overall scheduling process since the MAC scheduler does not perturb the 
scheduling decision made at IP level, and to limit or to prevent the loss in the MAC FIFOs.

\subsubsection{QoS tools}

In order to configure properly the QoS for every kind of application, different tools have been developed. Firstly, a QoS Server running in the satellite terminals is in charge of collecting QoS information on traffic flows and configuring accordingly the IP and MAC layers. Those information are provided by a QoS Agent for non-QoS aware applications or an enhanced SIP Proxy for QoS aware applications.

\subsubsection{QoS Agent}

Very few applications currently implemented are aware of the QoS provided by the underlying network. As the applications are rarely able to define their own requirements, a user-oriented solution, called « QoS Agent », has been defined in the SatIP6 project [2]. Implemented on the user terminal, the QoS Agent detects the outgoing traffic flows and thanks to a graphical interface listing them (cf. Figure 11), the user is able to assign one of the available QoS services and, through interactions with the QoS Server, to remotely configure the multi-field classifier in the RCST, for only one or for all the streams the application uses. In fact, each time a service is selected by the user for a given application, the QoS Agent sends to the QoS Server the concerned connections' list with the reference of the associated service using a dedicated transactional protocol. Using the received information, the QoS Server is able to tag and redirect these packets coming from the user terminal towards the appropriate requested IP service. If resources are not available, the QoS Agent is immediately informed.

\section{Fig. 11}

\subsubsection{Enhanced SIP Proxy}

An alternative solution to the QoS Agent is to automate the resources reservation and make it transparent to users who wouldn't be able to choose the most appropriate classes of service to the different flows (audio and video for example). To make that, the Session Initiation Protocol (SIP) has been chosen because of its growing success in both public (open source code) and private (company) fields. This mechanism allows to configure the QoS transparently by the analysis of the SIP messages exchanged between the applications. This analysis is made by an enhanced SIP Proxy (or QoS-aware SIP Proxy).

As shown in the Figure 12, the enhanced SIP Proxy intercepts the session descriptors included in SIP messages (INVITE and OK), deduces the characteristics of each media involved in the session and realize the reservations and releases with the QoS Server.

\section{Fig. 12}

Some additional functionalities are needed to realize that:

- An SDP analyser making the Proxy able to analyse the session descriptions

- A table of medias updated during the session establishment. The medias negotiated between the caller and the callee are identified by a call-ID.

- An SDP/Diffserv mapping

- A QoS module which realizes the resources reservation associated to each media with the QoS Server.

A QoS-Aware SIP proxy is deployed in each user LAN. This distributed architecture is well suited for an access or mesh topology based on a regenerative satellite because it answers the following two concerns: 
- scalability concerning flow QoS management in user LAN;

- session establishment delays: the number of round trips of session and QoS signalling on the satellite link are minimized.

\subsubsection{Interface with the QoS Server}

In both case, the exchange of resources reservation messages and resources release messages is performed through a TCP connection over IPv4/IPv6 to assure a reliable and ordered message delivery. The protocol used to communicate with the QoS Server is based on XML.

The structure of the messages exchanged by the QoS Agent or the enhanced SIP Proxy with the QoS Server is quite similar. In both case, it indicates the type of message (RSV, FREE) and the source address, the source port, the destination address and the destination port which are required to the identification of a specific connection. Then, in the QoS Agent case, it is necessary to precise the application, the PID of the application and the wished class of service and in the SIP Proxy case, the type of media for example.

\section{PLATINE outputs and control}

\subsection{Statistics, events and errors}

Additionally to aforementioned advantages, emulation provides interesting characteristics such as measurement points which would not be so easily accessible in real systems. Regarding our satellite emulator, statistics are computed at each superframe at the NCC/GW side, and at each frame at the RCST side (for both MAC layer and IP QoS layers).

These measurement points, as well events or errors, are handled by a distributed framework. Each emulation component embeds 3 agents in charge of collecting and gathering all these information with basic treatments (such as mean, max, ...) and sends them on a regular basis to centralized statistic, event and error controllers. These processes aggregate the data with optional processing (sliding window for example), store them in files according to the simulation and run numbers, and send them to a real time graphical display.

Statistics includes delay measurements, load measurements, queues, DAMA and congestion control information.

Figure 13 shows several measurement points at different levels on the RCST return link channel. Measurement points are located at ingress points, at egress points as well as inside of IP_QoS and MAC layers of the satellite network emulator. As a result, the DAMA algorithm effects can be observed at different levels of the system. So, when higher layers transmit traffic, we are able to measure the throughput per traffic category at IP layer ingress points and egress points. The throughput crossing the IP_QoS layer can be therefore characterized. Similarly, relevant IP QoS information extracted from the HDLB scheduler are available.

\section{Fig. 13}

The MAC layer behavior can also be analyzed through the study of the throughput at ingress and egress measurement points. Inside the MAC layer, the DAMA algorithm behavior can be evaluated. The evolution of capacity requests can be obtained for the NRT MAC service class in term of kbits/s and time-slot number. The DAMA efficiency can be directly obtained through the ratio between used and allocated time-slots.

We also benefit from statistics on the downlink channel, like the downlink traffic throughput received on the AIR interface by the RCST and the uplink traffic throughput sent on the AIR interface by the RCST. 
What's more, in order to study more thoroughly the DAMA algorithm efficiency in a configuration comprising numerous RCSTs, the NCC is supplemented with the ability to emulate virtual RCSTs. To do so, the MAC scheduler is able to replay virtual CRs and takes them into account in addition to CRs sent by individual emulated RCSTs. These virtual CRs may be obtained through past recorded logs or created from scratch. Moreover, this tool allows to generate background traffic to study congestion for instance.

\subsection{Managing the emulation testbed}

One of the classical problem of emulation testbeds is their poor user interface resulting to difficult configuration and management, especially in a distributed environment. PLATINE includes a new control interface (see Figure 14) to start and stop the emulation process on a configurable network topology. Moreover, it offers various services to retrieve the component status, to start network components such as multicast routers and to execute the JAVA display. Finally, it can distribute the complete software on a group of nodes in a LAN to simplify maintenance and tests.

\section{Fig. 14}

\section{Conclusion \& Further works}

This testbed is an important tool to evaluate DVB-S2/RCS system performance and in particular its impact on innovative network techniques. Providing the user with several kinds of measurement, traffic flows and results analysis tools, it is an interesting way to investigate how to configure and use such systems.

This testbed is efficient enough to support various kinds of experimentation, dealing with multimedia user applications for instance. It may be enhanced in order to implement mechanisms such GSE. Thanks to the software design of the testbed, enhancements may be done easily. Any interested user is clearly welcome.

Acknowledgements

This work is supported by the IST FP6 SATSIX project, funded by European Commission (EC). The financial contribution of the EC towards this project is greatly appreciated.

Reference

[1] IST SATSIX Project (Contract IST-2004-26950), http://www.ist-satsix.org

[2] IST SATIP6 Project (Contract IST-2001-34344), http://satip6.tilab.com

[3] Margouilla c++ Runtime : http://cqsoftware.free.fr/margouilla

[4] ETSI TR102157 v1.1.1. ETSI TC SES; Broadband Satellite Multimedia; IP Interworking over satellite; Performance, Availability and Quality of Service, July 2003

[5] A.Pietrabissa, T.Inzerilli, O.Alphand, P.Berthou, E.Fromentin, T.Gayraud, F.Lucas. Validation of a QoS architecture for DVB/RCS satellite networks via a hardware demonstration platform, Computer Networks Journal, Issue 6, Volume 49, pp. 797-815, December 2005.

[6] O. Alphand, P.Berthou, T.Gayraud, S. Combes, QoS Architecture over DVBRCS satellite networks in a NGN framework, GLOBECOM 2005, St-Louis USA, December 2005.

[7] O. Alphand, P. Berthou, T. Gayraud, F. Nivor, S. Combes, C. Baudoin, PLATINE: DVB-S/RCS Testbed for Next Generation Satellite Networks, ASMS 2006, Munich Germany, May 2006.

[8] http://luxik.cdi.cz/ devik/qos/htb/manual/theory.htm 
[9] G. Fairhurst, B. Collini-Nocker, Unidirectional Lightweight Encapsulation (ULE) for Transmission of IP Datagrams over an MPEG-2 Transport Stream (TS), RFC 4326, December 2005

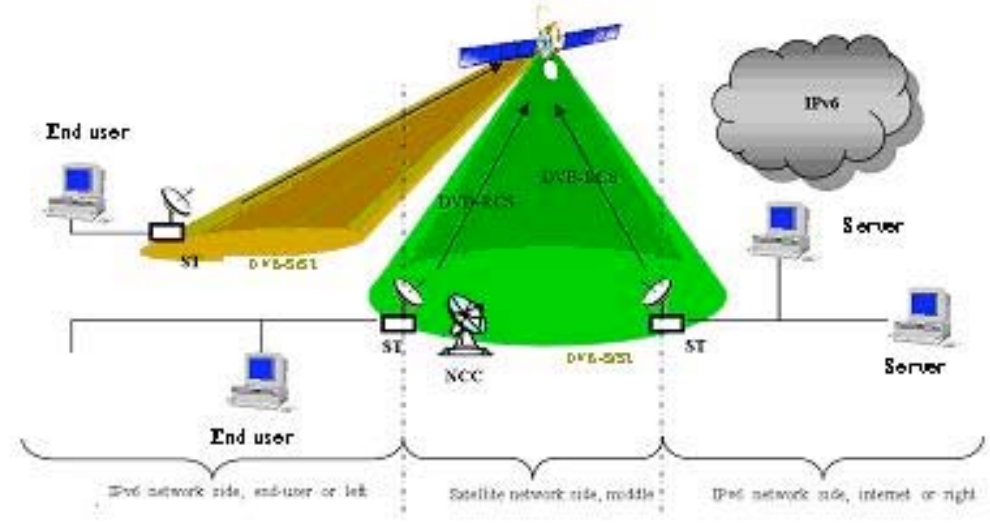

Figure 1: DVB-S2/RCS architecture

\begin{tabular}{|c|c|c|}
\hline \multicolumn{3}{|c|}{ IP } \\
\hline AALS & ULE & ULE \\
\hline ATM & MPEG2-TS & MPEG2-TS \\
\hline \multicolumn{2}{|c|}{ DVB-RCS } & DVB-S2 \\
\hline \multicolumn{3}{|c|}{$\llbracket$} \\
Uplink & Downlink
\end{tabular}

Figure 2: PLATINE protocol stack (star topology) 


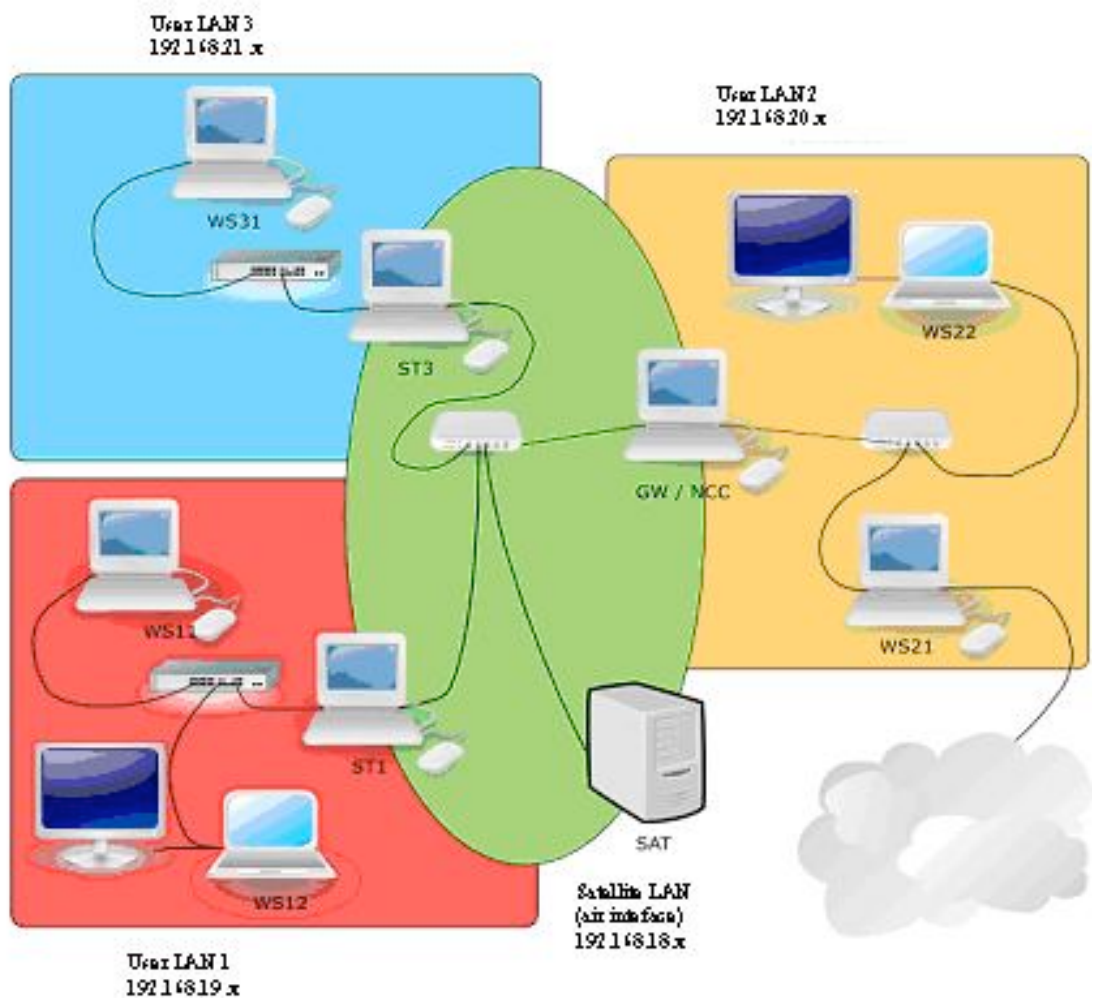

Figure 3: Full PLATINE testbed

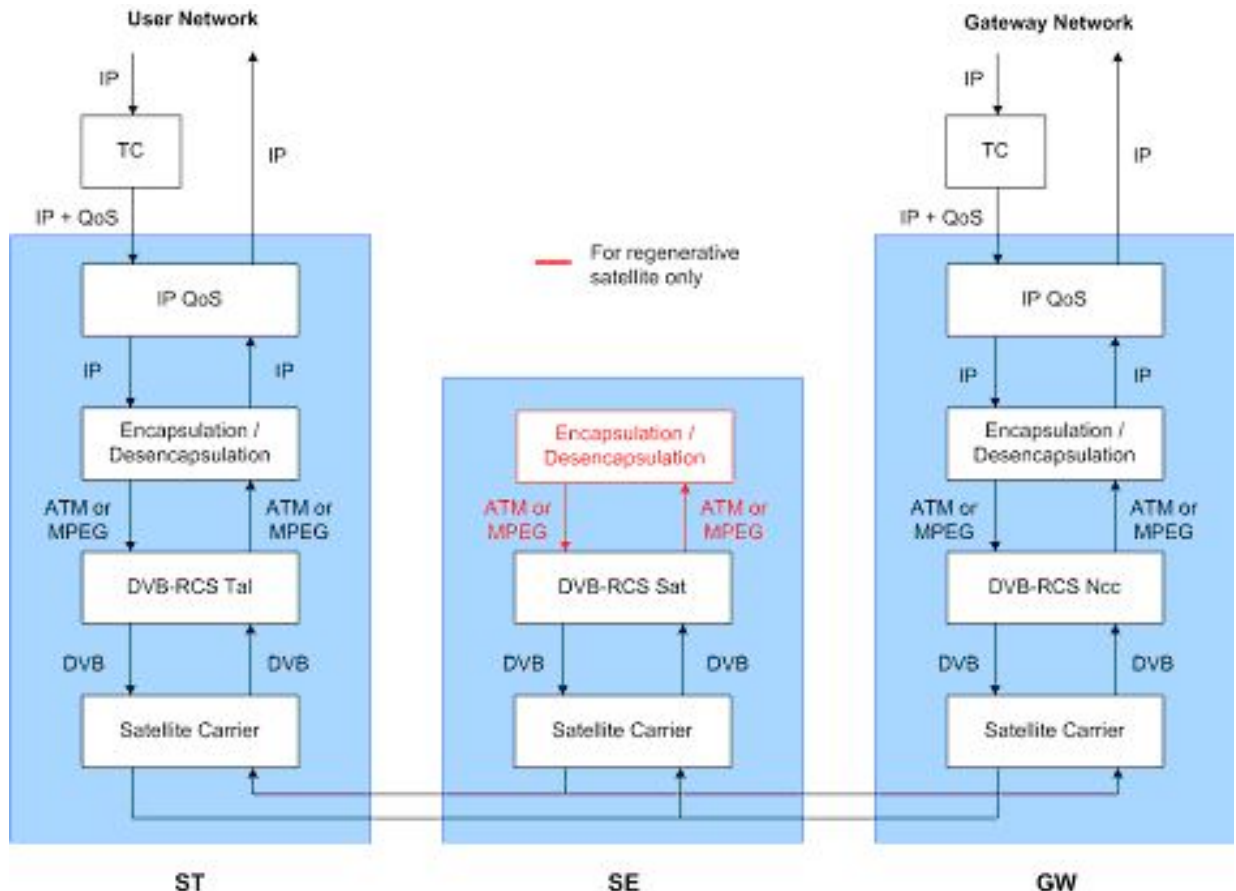

Figure 4: PLATINE emulation platform architecture 


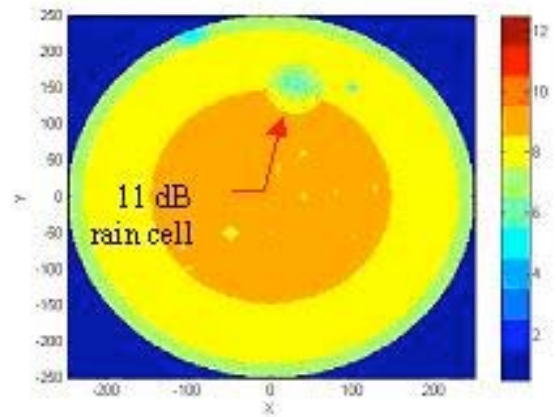

Figure 5: Return link scenario with individual terminals: spatial repartition of the DRA schemes in the spot at the beginning of the simulation

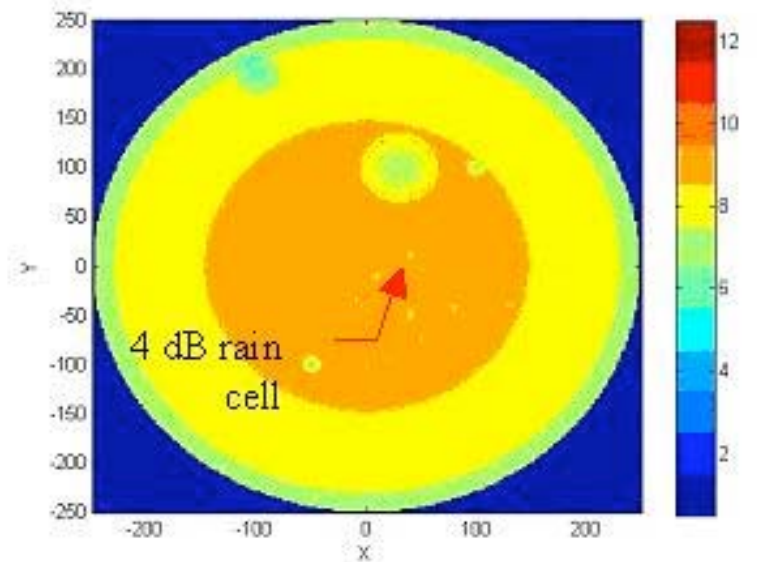

Figure 6: Return link scenario with individual terminals: spatial repartition of the DRA schemes in the spot at the end of the simulation

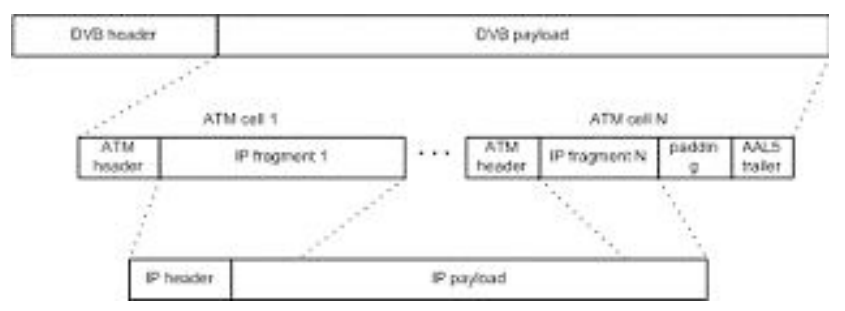

Figure 7: AAL5/ATM encapsulation scheme

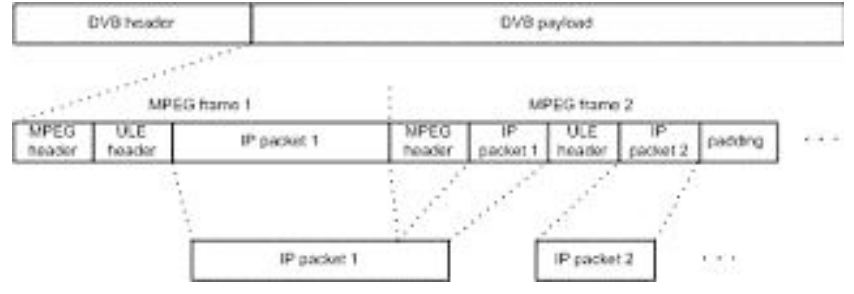

Figure 8: ULE/MPEG-2 TS encapsulation scheme 


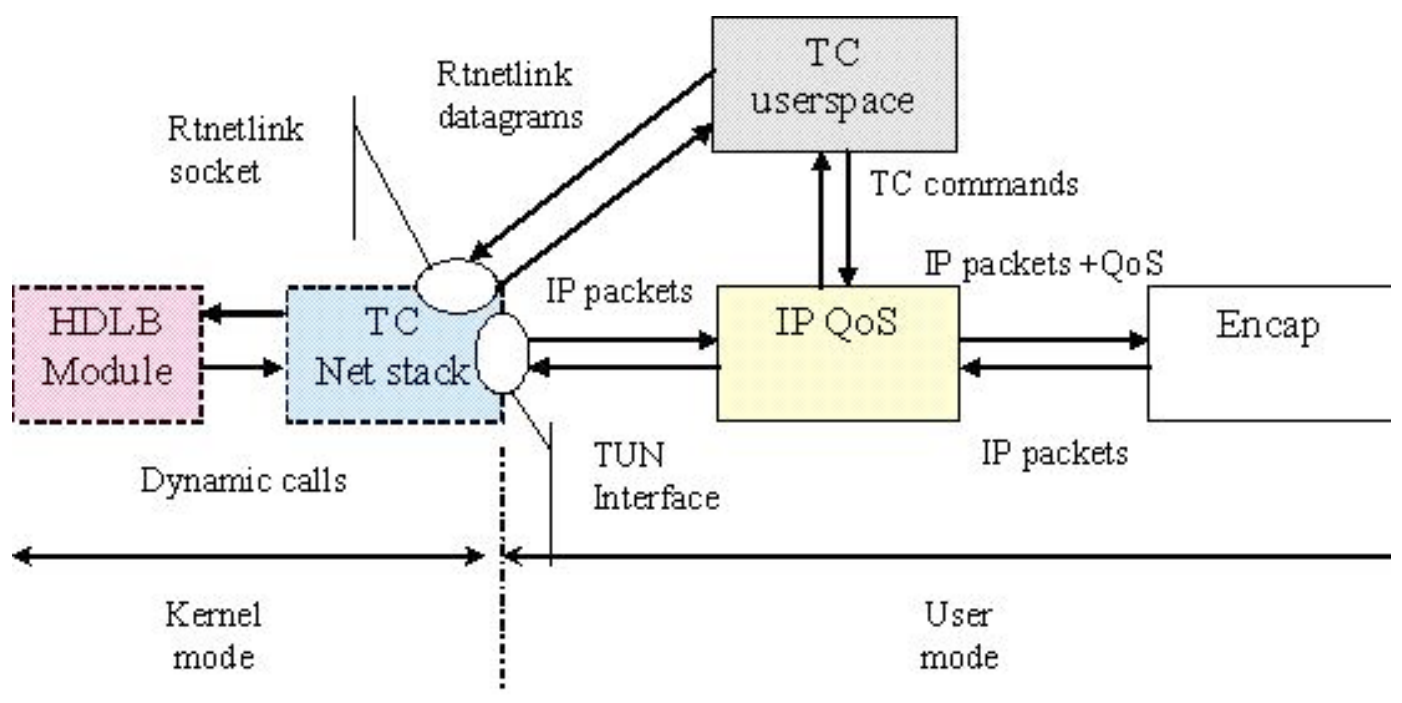

Figure 9: IP QoS architecture

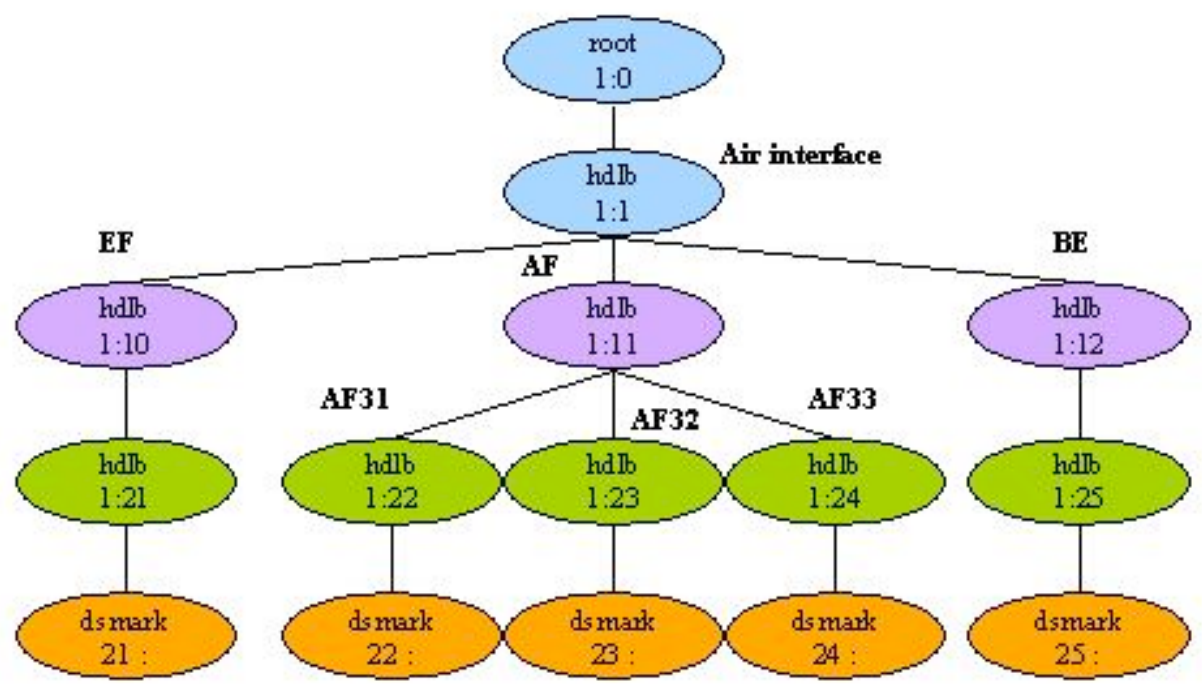

Figure 10: HDLB overview

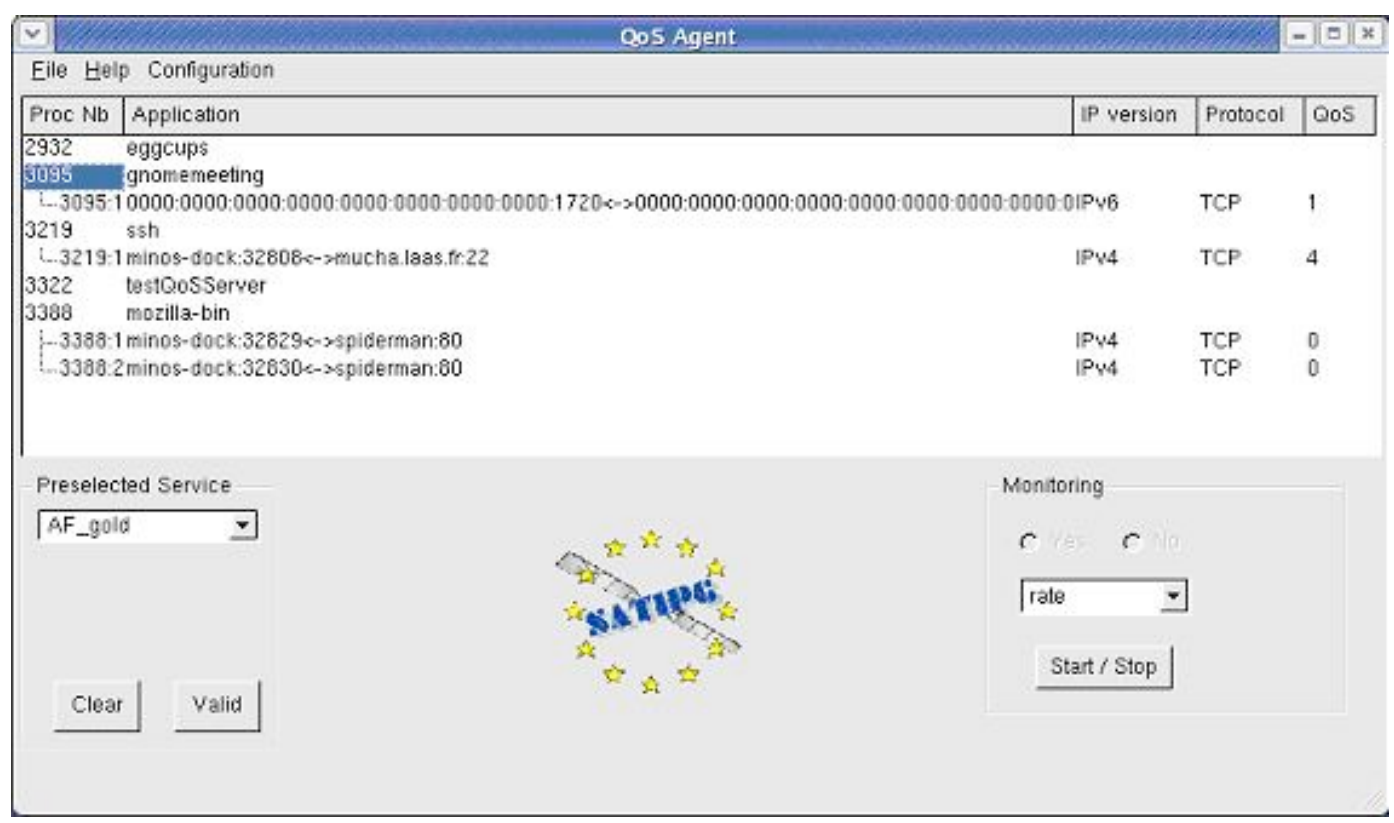


Figure 11: QoS Agent graphical interface

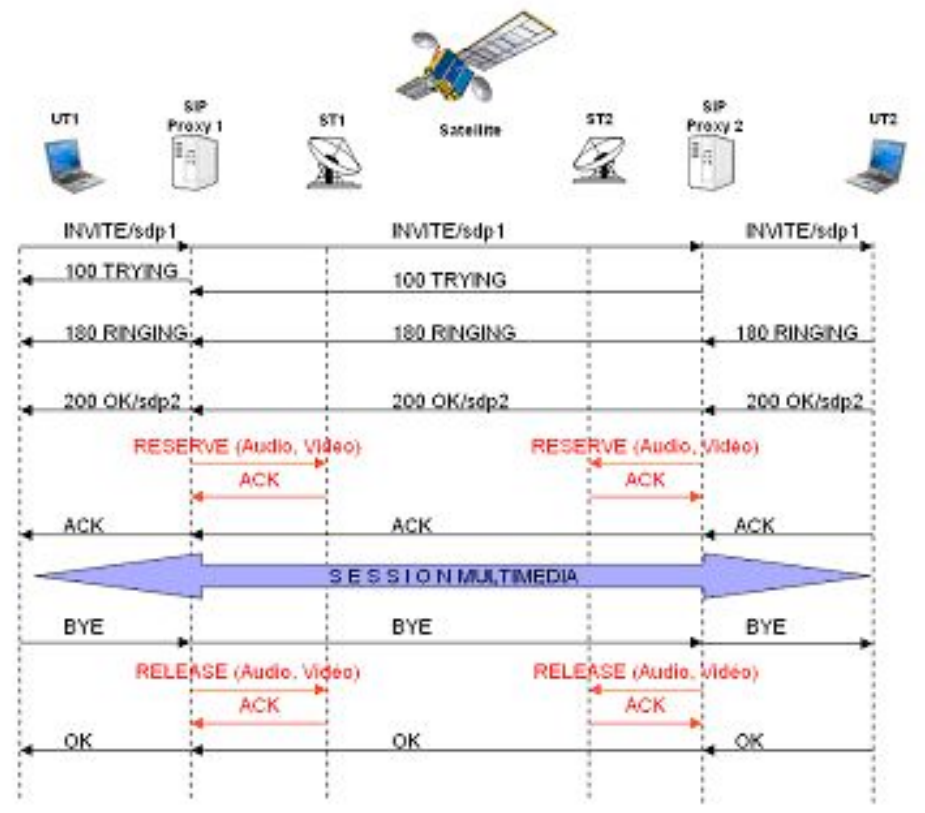

Figure 12: Establishment of a SIP session with QoS

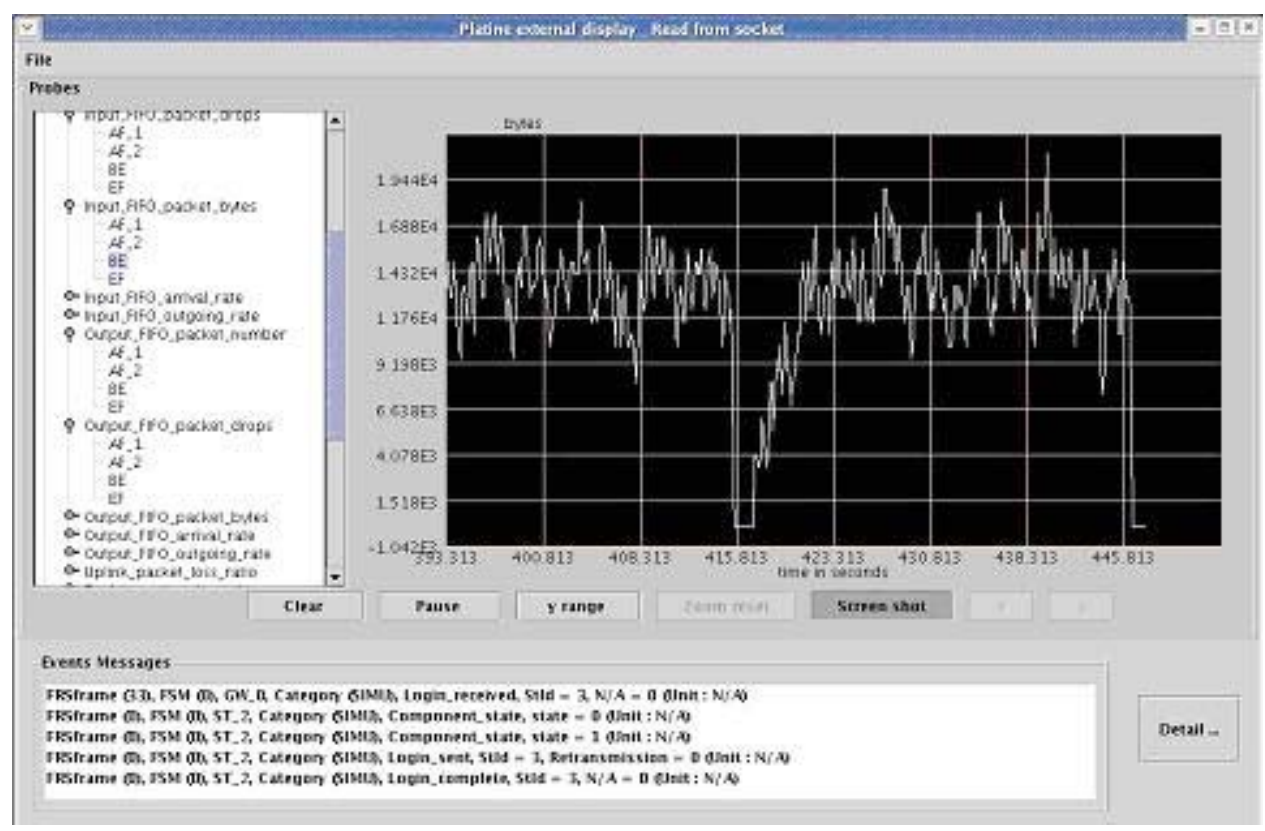

Figure 13: PLATINE statistics tool

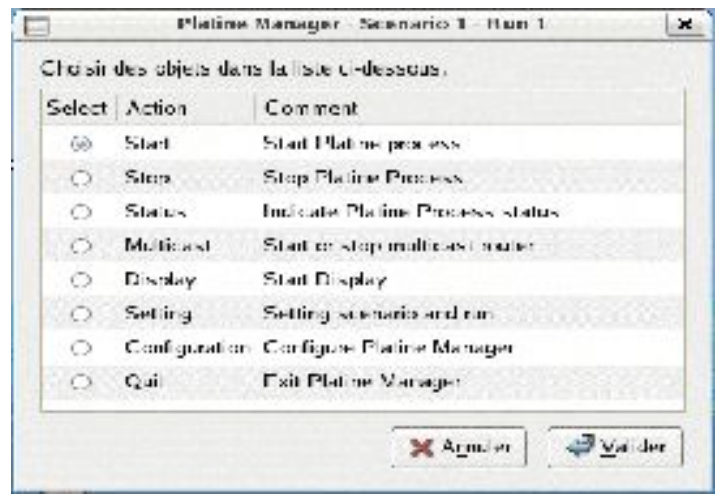

Figure 14: Control User Interface 
